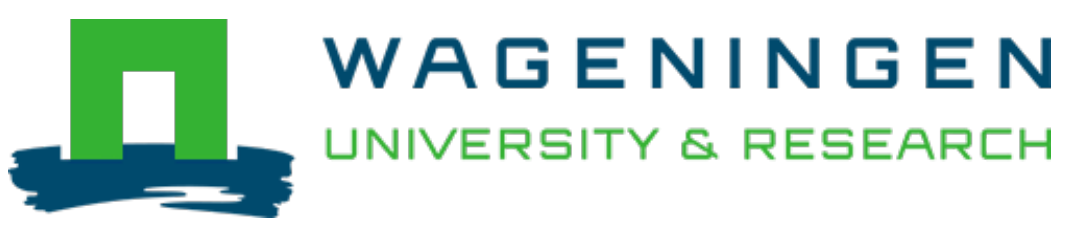

\title{
Does food intake mediate the association between mindful eating and change in depressive symptoms?
}

\author{
Public Health Nutrition \\ Winkens, L.H.H.; Elstgeest, L.E.M.; Strien, T.; Penninx, B.W.J.H.; Visser, M. et al \\ https://doi.org/10.1017/S1368980019003732
}

This article is made publicly available in the institutional repository of Wageningen University and Research, under the terms of article $25 \mathrm{fa}$ of the Dutch Copyright Act, also known as the Amendment Taverne. This has been done with explicit consent by the author.

Article 25 fa states that the author of a short scientific work funded either wholly or partially by Dutch public funds is entitled to make that work publicly available for no consideration following a reasonable period of time after the work was first published, provided that clear reference is made to the source of the first publication of the work.

This publication is distributed under The Association of Universities in the Netherlands (VSNU) 'Article $25 \mathrm{fa}$ implementation' project. In this project research outputs of researchers employed by Dutch Universities that comply with the legal requirements of Article $25 \mathrm{fa}$ of the Dutch Copyright Act are distributed online and free of cost or other barriers in institutional repositories. Research outputs are distributed six months after their first online publication in the original published version and with proper attribution to the source of the original publication.

You are permitted to download and use the publication for personal purposes. All rights remain with the author(s) and / or copyright owner(s) of this work. Any use of the publication or parts of it other than authorised under article $25 \mathrm{fa}$ of the Dutch Copyright act is prohibited. Wageningen University \& Research and the author(s) of this publication shall not be held responsible or liable for any damages resulting from your (re)use of this publication.

For questions regarding the public availability of this article please contact openscience.library@wur.nl 


\title{
Does food intake mediate the association between mindful eating and change in depressive symptoms?
}

\author{
LHH Winkens $^{1,2, *}$, LEM Elstgeest ${ }^{2}$, T van Strien ${ }^{2,3}$, BWJH Penninx ${ }^{4}, M$ Visser $^{2}$ and \\ IA Brouwer ${ }^{2}$ \\ 'Consumption and Healthy Lifestyles Chair Group, Wageningen University \& Research, Hollandseweg 1, 6706 KN \\ Wageningen, The Netherlands: ${ }^{2}$ Department of Health Sciences, Faculty of Science, Amsterdam Public Health \\ Research Institute, Vrije Universiteit Amsterdam, Amsterdam, The Netherlands: ${ }^{3}$ Behavioural Science Institute, \\ Radboud University, Nijmegen, The Netherlands: ${ }^{4}$ Department of Psychiatry, Amsterdam Public Health Research \\ Institute, Amsterdam UMC, Vrije Universiteit Amsterdam, Amsterdam, The Netherlands
}

Submitted 6 November 2018: Final revision received 30 July 2019: Accepted 19 August 2019

\begin{abstract}
Objective: To examine mediation by (i) diet quality and (ii) diet quantity in the associations of mindful eating domains with 3-year change in depressive symptoms.

Design: Depressive symptoms were measured with the Center for Epidemiologic Studies Depression scale at baseline and 3-year follow-up. Four mindful eating domains (Focused Eating; Eating in response to Hunger and Satiety Cues; Eating with Awareness; Eating without Distraction) were measured with the Mindful Eating Behavior Scale. Food intake was measured with a 238-item FFQ. Diet quality was defined as the Mediterranean Diet Score (MDS). Diet quantity was defined as total energy intake $(\mathrm{kcal} / \mathrm{d} ; 1 \mathrm{kcal}=4 \cdot 184 \mathrm{~kJ})$. Mediation analyses with percentile-corrected bootstrap confidence intervals were conducted to calculate indirect effects.

Setting: Longitudinal Aging Study Amsterdam.

Participants: Adults aged 55 years or above ( $n$ 929).

Results: Diet quality (MDS) did not mediate associations of any of the four mindful eating domains with change in depressive symptoms. In contrast, total energy intake did mediate the associations with change in depressive symptoms for the mindful eating domains Eating with Awareness (indirect effects fully adjusted models: $B=-0.014,95 \%$ CI $-0.037,-0.002)$ and Eating without Distraction ( $B=-0.013,95 \% \mathrm{CI}-0.033,-0.001)$, but not for the other two domains. Post hoc multiple mediation analyses showed similar results.

Conclusions: Higher scores on two mindful eating domains were associated with a decrease in depressive symptoms through lower total energy intake. Diet quantity, but not diet quality, could be a possible underlying mechanism in the associations between mindful eating and change in depressive symptoms.
\end{abstract}

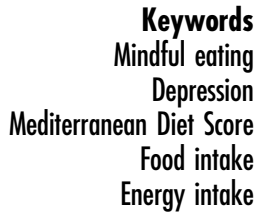

Keywords

dful eating

Diet Score

Energy intake
Depression is a highly prevalent mental disorder influenced by a complex interplay of factors ${ }^{(1)}$. Mindfulness-based interventions led to reductions in depressive symptoms ${ }^{(2-5)}$. Recently, more evidence has been gathered on the associations of lifestyle factors, such as quality of food intake and BMI, with depression ${ }^{(6-9)}$. As mindfulness-based interventions with an eating component were much more effective in reducing maladaptive eating behaviours compared with interventions without a mindful eating component ${ }^{(10,11)}$, previous research started to examine associations between mindful eating and mental well-being. Mindful eating can be defined as 'eating with awareness and attention' and was associated with (change in) depressive symptoms ${ }^{(12,13)}$. Underlying mechanisms are not clear yet. Could food intake potentially mediate the association between mindful eating and change in depressive symptoms?

Both quantity and quality of food intake may be associated with mindful eating and depression. Food quantity is the total weight of the foods consumed and is generally, but not necessarily, reflected in the total amount of energy 
consumed. Food quality is the extent to which the diet meets specific dietary recommendations. The quantity and quality of the diet are thus two different concepts and are not necessarily related. However, when energy intake is high, a person is more likely to meet a specific food group (e.g. fish) requirement. Gougeon et al. found in a community-dwelling older population that dietary patterns were not related to depression, whereas energy intake was $^{(14)}$. This suggests that both can be independently associated with depression. Food intake might be associated with depression through its influence on different physiological processes that are involved in the development of depression, such as hypothalamic-pituitary-adrenal axis disturbances, inflammation and neurotransmitter imbalances ${ }^{(15,16)}$.

Mindful eating was the underlying mechanism in the association between higher general mindfulness and smaller serving size of energy-dense foods ${ }^{(17)}$. In a review on mindfulness interventions for weight loss and eating behaviours, findings from three out of four studies on attendance to the sensory properties of food did not find significant reductions in immediate amount of food consumed ${ }^{(18)}$. However, significant reductions in later amounts of food intake were found in a normal-weight and an overweight/obese population $^{(18)}$. This finding was replicated for later snack intake in a sample of normal-weight men and women ${ }^{(19)}$, while there was no reduced later snack intake in other experimental studies ${ }^{(20,21)}$. In patients with type 2 diabetes mellitus, a mindful eating intervention led to reduced energy intake immediately following the intervention and at the 3 -month follow-up ${ }^{(22)}$. In pregnant women, higher awareness for eating was associated with higher intake of fruit and vegetables ${ }^{(23)}$.

There is very limited knowledge on mechanisms of action for the associations between mindful eating and food intake, but some potential explanations can be given. A reduction in eating automaticity and a prioritization of the amount of pleasure obtained from food instead of reaching satiation could explain the associations between mindful eating and decreased energy intake ${ }^{(18)}$. Increased interoceptive awareness (the ability to recognize and respond to internal states such as emotions, hunger and satiety) was associated with higher reliance on hunger and satiety cues $^{(24,25)}$, and mediated the association between reliance on hunger and satiety cues and $\mathrm{BMI}^{(24)}$. This suggests that mindful eating might influence habitual food intake through an increase in interoceptive awareness. Another potential explanation is that mindful eating reduces cravings for food ${ }^{(26,27)}$, which is associated with higher intake of unhealthy food products ${ }^{(28)}$. Finally, mindful eating could lead to increased self-regulation, which then leads to a more healthy food intake ${ }^{(29)}$.

Mindful eating may thus be related to food intake, but research is scarce and findings are mixed. The majority of the studies are not conducted in general population samples. Furthermore, those studies investigated specific dietary aspects, such as intake of high-energy foods, serving size and energy consumed after experimental manipulations, whereas habitual food intake measured over a longer time period may better reflect actual intake. In the last decades, nutritional research has shifted its focus from nutrients and foods towards dietary patterns ${ }^{(30)}$. The Mediterranean Diet Score (MDS) is a useful tool to measure adherence to a healthy $\operatorname{diet}^{(31,32)}$ and is associated with a lower risk of depression ${ }^{(33-35)}$. Higher energy intake was related to more depressive symptoms in adolescents ${ }^{(36)}$, but in older samples lower energy intake may also be predictive of depression due to declining general health ${ }^{(37)}$.

The aim of the present study was to investigate mediation of associations of mindful eating domains (Focused Eating; Eating in response to Hunger and Satiety Cues; Eating with Awareness; Eating without Distraction) with 3-year change in depressive symptoms by (i) diet quality as indexed by the MDS and (ii) diet quantity as indexed by total energy intake. Hypotheses were that mindful eating would be related to higher diet quality and lower diet quantity and that this would be related to lower depressive symptoms. There were no specific hypotheses regarding the different mindful eating domains.

\section{Methods}

\section{Participants and procedure}

Data were collected within the Longitudinal Aging Study Amsterdam (LASA), an ongoing cohort study in a representative sample of Dutch older adults aged 55 years or above, which started in 1992. The sampling and data collection procedures have been described in detail elsewhere ${ }^{(38,39)}$. Ethical approval for LASA was given by the Medical Ethics Committee of the VU University Medical Center Amsterdam and all participants provided written informed consent.

Mindful eating and food intake were measured in the ancillary 'LASA Nutrition and Food-related Behaviour study' that was conducted between the autumn of 2014 and the spring of $2015^{(40)}$. Depressive symptoms and antidepressant use were measured in the regular measurement waves of LASA: baseline data were measured in either 2011-2012 (cohorts 1 and 2) or 2012-2013 (cohort 3); and follow-up data were collected in 2015-2016. By using the depressive symptoms score at the follow-up measurement as outcome variable and adjusting analyses for baseline depressive symptoms, the outcome was interpreted as 'change in depressive symptoms'. However, it should be noted that mindful eating and food intake were measured in-between this follow-up period, thereby making strict longitudinal interpretation of associations impossible. 


\section{Measures}

\section{Depressive symptoms}

The Center for Epidemiologic Studies Depression scale $(\mathrm{CES}-\mathrm{D})^{(41)}$ was used to measure depressive symptoms in the past week and was administered in face-to-face interviews. The scale has twenty items with a 4-point scale ranging from 0 (rarely or none of the time; less than $1 \mathrm{~d}$ ) to 3 (most or almost all the time; 5-7 d). A higher score indicates a higher level of depressive symptoms. Cronbach's $\alpha$ of the CES-D was 0.87 (cohorts 1 and $2 ; n$ 494) and 0.85 (cohort 3; $n 435)$ at baseline, and 0.85 at the follow-up measurement. The continuous CES-D score at the follow-up measurement was used as outcome variable, while controlling for the baseline CES-D score.

People with antidepressant use at baseline and/or the follow-up measurement were excluded from all analyses. Data on antidepressant use were retrieved by asking about medicine use. Medication names were recoded into Anatomical Therapeutic Chemical codes, which were used to define the use of antidepressants (yes/no) ${ }^{(42)}$.

\section{Mindful eating domains}

The Mindful Eating Behavior Scale (MEBS) ${ }^{(40)}$ was used to measure the level of four different domains of mindful eating: (i) Focused Eating (five items, e.g. 'I notice how my food looks'); (ii) Eating in response to Hunger and Satiety Cues (five items, e.g. 'I trust my body to tell me when to eat'); (iii) Eating with Awareness (three items, e.g. 'I eat something without being really aware of it', reversed item); and (iv) Eating without Distraction (four items, e.g. 'I multitask when I am eating', reversed item). Answer options ranged from 1 ('never') to 5 ('very often'). Higher scores indicate a higher level of mindful eating. Cronbach's $\alpha$ of the mindful eating domains was 0.85 for Focused Eating, 0.90 for Eating in response to Hunger and Satiety Cues, 0.82 for Eating with Awareness and 0.70 for Eating without Distraction.

\section{Food intake}

Food intake was assessed using a 238-item semi-quantitative FFQ with a reference period of 4 weeks, that was developed for the HEalthy LIfe in an Urban Setting study (HELIUS) ${ }^{(43)}$. This FFQ is an adapted version of an existing validated FFQ for a Dutch population ${ }^{(44)}$. To calculate nutrient intakes, all food items were linked to a nutrient database that was based on the Dutch Food Composition Database ${ }^{(45)}$.

Energy intake was calculated by summing the consumed kilocalories of all food items per day to achieve the total amount $(\mathrm{kcal} / \mathrm{d} ; 1 \mathrm{kcal}=4 \cdot 184 \mathrm{~kJ})$.

The MDS, an a priori, theoretically defined dietary pattern assessing diet quality, provides a measure of the adherence to the Mediterranean diet, which is characterized by high intakes of cereals, legumes, fruit, vegetables, olive oil and fish. The MDS was found to be a useful tool to measure adherence to a healthy diet ${ }^{(46,47)}$. Previous studies showed associations between Mediterranean-like dietary patterns and health benefits, also in non-Mediterranean countries $^{(48)}$. The MDS was calculated by summing scores from 0 to 5 based on the intake in weekly or daily servings of eleven food groups ${ }^{(24)}$. The total score ranged from 0 to 55. A higher score indicates a healthier diet.

\section{Confounders}

Confounders in different models were sex, age, educational level, smoking status, physical activity level, alcohol consumption and BMI, because demographic and lifestyle factors have shown to be related to both mindful eating and depression and might therefore bias the associations under study. These variables were measured at the baseline measurement (2011-2013).

Sex and age were derived from the municipal registries.

Educational level was self-reported and categorized into low (none, elementary school), medium (secondary education, lower and intermediate vocational training) and high (higher vocational training, college and university education).

Smoking status (never/former/current) was selfreported.

Physical activity was measured using the validated LASA Physical Activity Questionnaire (LAPAQ) ${ }^{(49)}$. Frequency and duration of walking outdoors, bicycling, light and heavy household activities and sports in the past 2 weeks were asked. Total time in minutes per day spent on these activities was calculated.

Alcohol consumption was assessed by asking respondents about the number of days per week they drank alcohol and the number of alcoholic drinks on these days ${ }^{(50)}$. The number of alcoholic consumptions per week was calculated.

BMI $\left(\mathrm{kg} / \mathrm{m}^{2}\right)$ was calculated by dividing measured weight by the square of measured height. Weight was measured to the nearest $0 \cdot 1 \mathrm{~kg}$ using a calibrated bathroom scale (Seca model 100; Lameris, Utrecht, The Netherlands). Corrections were made to adjust the measured body weight for clothing, shoes or a corset $(-1 \mathrm{~kg}$ for one of those elements and $-2 \mathrm{~kg}$ for more than one) when people did not wear underclothing only. Height was measured to the nearest $0.001 \mathrm{~m}$ using a stadiometer. Corrections were made to adjust the measured height for shoes $(-1 \mathrm{~cm})$ when people did not take their shoes off.

\section{Statistical analysis}

The proposed associations (mediation and confounding variables) are depicted in Fig. 1. All analyses were conducted using the statistical software package IBM SPSS Statistics version 23.0. People were included who had data on depressive symptoms at both the baseline and followup measurement. People who did not fill out the questionnaire of the 'LASA Nutrition and Food-related Behaviour study' themselves or who had missing values on one of more of the used variables were excluded from the analyses. For the calculation of the MDS and total energy 
(a)

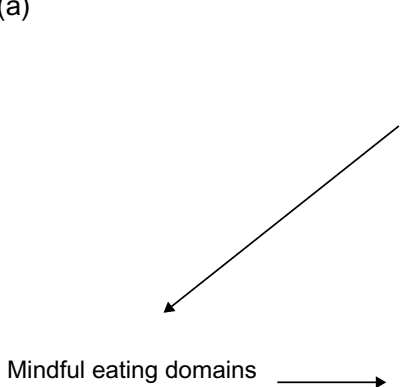
(2014-2015)

(b)

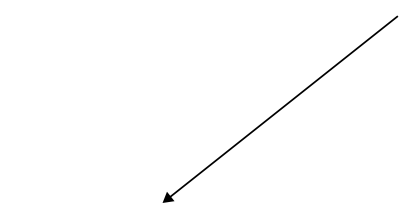

Mindful eating domains (2014-2015)
Depressive symptoms T1

Sex

Age

Educational level

Smoking status

Physical activity level

Total energy intake BMI

(2011-2013)

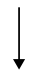

Food intake quality: MDS

(2014-2015)

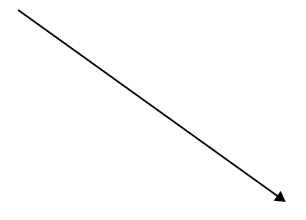

Depressive symptoms T2

(2015-2016)

Depressive symptoms T1

Sex

Age

Educational level

Smoking status

Physical activity level

Alcohol consumption

(2011-2013)

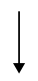

Food intake quantity:

Total energy intake

(kcal/d)

(2014-2015)
Depressive symptoms T2

(2015-2016)

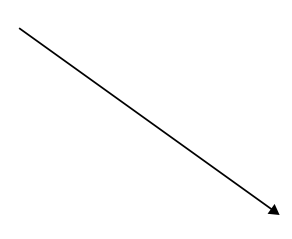

Fig. 1 Directed acyclic graph for proposed associations: (a) mediation by diet quality (Mediterranean Diet Score; MDS) in the association between mindful eating and 3-year change in depressive symptoms; (b) mediation by diet quantity (total energy intake; kcal/d; $1 \mathrm{kcal}=4.184 \mathrm{~kJ}$ ) in the association between mindful eating and 3-year change in depressive symptoms (T1/T2, baseline/follow-up of the Longitudinal Aging Study Amsterdam)

intake, $>10$ missing values on the FFQ questions was used as a criterion for exclusion of participants. People with extreme values for energy intake based on sex-specific cut-offs were also excluded $(<3347 \cdot 2 \mathrm{~kJ}(<800 \mathrm{kcal})$ or $>16736 \mathrm{~kJ}(>4000 \mathrm{kcal})$ for men and $<2092 \mathrm{~kJ}$ ( $<500 \mathrm{kcal})$ or $>14644 \mathrm{~kJ}$ (>3500 kcal) for women) ${ }^{(51)}$. People with antidepressant use at baseline and/or the follow-up measurement were excluded because of possible distortion of the CES-D scores.

Descriptive statistics were calculated to describe the sample.

To test whether MDS and total energy intake mediated the associations of mindful eating domains with change in depressive symptoms, percentile-corrected bootstrap confidence intervals for the indirect effects were calculated in separate mediation models. The PROCESS macro of Hayes $^{(52)}$ was used to test the mediation role of MDS and total energy intake on the association of a mindful eating domain and change in depressive symptoms. Change in depressive symptoms was defined as depression at $\mathrm{T} 2$ over and above depression at T1 (by performing the regression of depression at $\mathrm{T} 2 v$. depression at $\mathrm{T} 1$ in the analysis). This analysis was conducted for each of the four mindful eating domains. Bootstrapping with 5000 samples was used. Results were considered significant if the upper and lower bound of the percentile bootstrap $95 \%$ confidence interval did not contain zero.
To analyse changes in depressive symptoms, all analyses were adjusted for baseline depressive symptoms (model 1). Analyses were additionally adjusted for sex, age, educational level, smoking status and physical activity level (model 2). When assessing MDS as mediator, analyses were additionally adjusted for total energy intake (model 3). When assessing total energy intake as mediator, analyses were additionally adjusted for alcohol consumption (model 3). It was also tested if any of the associations changed when the analyses were additionally adjusted for BMI (model 4).

For the significant mediation analyses, sensitivity analyses were conducted using the 'medsens' command of the $\mathrm{R}$ package 'mediation', which calculates true indirect and direct effects for different values of the sensitivity parameter $(\rho)^{(53)}$. The sensitivity analysis quantifies the degree of violation of sequential ignorability based on the correlation between the error terms of the mediator and of the outcome ${ }^{(54)}$. Bootstrapping with 5000 samples was used.

\section{Results}

\section{Analytical sample}

Complete baseline data on depressive symptoms were available for 2268 people (1261 from cohorts 1 and 2; 1007 from cohort 3), of whom 1684 people had complete 
CES-D data on the follow-up measurement as well. Of them, 1302 people had data on the ancillary 'LASA Nutrition and Food-related Behaviour study'. People were excluded who did not fill out the questionnaire of this ancillary study themselves ( $n$ 103), who had one or more missing values on the MEBS ( $n 59)$ or missing values on any of the confounding variables ( $n 84)$. People with extreme values for energy intake based on sex-specific cut-offs ( $n$ 16) were excluded, as well as people with $>10$ missing values on the FFQ ( $n 3)$. People with antidepressant use at baseline and/or follow-up ( $n 75)$ as well as people with missing values on the questions on antidepressant use ( $n$ 33) were also excluded, which resulted in an analytical sample of 929 people. The characteristics of this sample are described in Table 1. Pearson correlations between variables are shown in Table 2.

\section{Mediation analyses: dietary quality}

Significant direct effects were found for the domains Focused Eating, Eating with Awareness and Eating without Distraction with change in depressive symptoms, but not for the domain Eating in response to Hunger and Satiety Cues (see Figs 2 and 3). However, mediation can exist and be tested even in the absence of such an overall significant association ${ }^{(30,31)}$. We therefore proceeded to examine whether food intake mediated the association between Eating in response to Hunger and Satiety Cues and depressive symptoms.

The indirect effects of the adjusted models of the mediation analyses of the MDS in the associations of the mindful eating domains with depressive symptoms are shown in Table 3. There was no mediation by the MDS for any of the four mindful eating domains in all of the adjusted models (indirect effects fully adjusted models: $B=0.002$, $95 \%$ CI -0.015 , 0.021 for Focused Eating; $B=-0.001$, $95 \%$ CI -0.006 , 0.003 for Eating in response to Hunger and Satiety Cues; $B=0.0001,95 \%$ CI $-0.016,0.015$ for Eating with Awareness; $B=0.001,95 \%$ CI $-0.004,0.005$ for Eating without Distraction).

In Fig. 2, the coefficients of the various paths of the mediation models with MDS as mediator are shown. The MDS was not significantly associated with change in depressive symptoms when controlling for any of the mindful eating domains. The mindful eating domains Focused Eating and Eating with Awareness were significantly associated with the MDS: in the fully adjusted models, a 1 point increase in Focused Eating was associated with an increase of 0.25 points on the MDS, and a 1 point increase in Eating with Awareness was associated with an increase of $0 \cdot 16$ points on the MDS, which is indicative of a healthier diet. Changes in associations when additionally adjusting for BMI (model 4) were negligible (e.g. association between Focused Eating and the MDS model 4: $B=0 \cdot 24)$. Inclusion of people with extreme values on energy intake ( $n$ 16) did not change any of these results.
Table 1 Baseline characteristics of the study sample of Dutch people aged 55 years or above from the Longitudinal Aging Study Amsterdam ( $n$ 929)

\begin{tabular}{|c|c|c|c|}
\hline & Mean or $\%$ & SD & $n$ \\
\hline Age (years) & $66 \cdot 7$ & $7 \cdot 6$ & - \\
\hline Sex (\% female) & $49 \cdot 0$ & - & 455 \\
\hline \multicolumn{4}{|l|}{ Education (\%) } \\
\hline Low & $10 \cdot 9$ & - & 101 \\
\hline Medium & $57 \cdot 5$ & - & 534 \\
\hline High & 31.6 & - & 294 \\
\hline Physical activity $(\min / \mathrm{d})$ & $160 \cdot 0$ & $107 \cdot 1$ & - \\
\hline Alcohol use (glasses/week) & $10 \cdot 2$ & $10 \cdot 5$ & - \\
\hline \multicolumn{4}{|l|}{ Smoking (\%) } \\
\hline Never & $28 \cdot 1$ & - & 261 \\
\hline Former & $61 \cdot 7$ & - & 573 \\
\hline Current & $10 \cdot 2$ & - & 95 \\
\hline BMI $\left(\mathrm{kg} / \mathrm{m}^{2}\right)$ & $26 \cdot 9$ & $4 \cdot 2$ & - \\
\hline $\begin{array}{l}\text { Depressive symptoms, } \\
\text { CES-D score }\end{array}$ & $5 \cdot 0$ & $2 \cdot 0-9 \cdot 0$ & - \\
\hline \multicolumn{4}{|l|}{ Mindful eating, MEBS score } \\
\hline Focused Eating & $20 \cdot 1$ & $3 \cdot 3$ & - \\
\hline $\begin{array}{l}\text { Eating in response to } \\
\text { Hunger and Satiety Cues }\end{array}$ & $15 \cdot 4$ & $5 \cdot 2$ & - \\
\hline Eating with Awareness & $13 \cdot 0$ & $2 \cdot 1$ & - \\
\hline Eating without Distraction & $15 \cdot 5$ & $2 \cdot 7$ & - \\
\hline MDS & $32 \cdot 9$ & 4.7 & - \\
\hline Total energy intake $(\mathrm{kJ} / \mathrm{d})$ & 8669 & 2360 & - \\
\hline Total energy intake $(\mathrm{kcal} / \mathrm{d})$ & 2072 & 564 & - \\
\hline
\end{tabular}

CES-D, Center for Epidemiologic Studies Depression scale; MEBS, Mindful Eating Behavior Scale; MDS, Mediterranean Diet Score.

*The median score and interquartile range of depressive symptoms are presented because of a skewed distribution.

\section{Mediation analyses: diet quantity}

The indirect effects of the adjusted models of the mediation analyses of total energy intake in the associations of the mindful eating domains with depressive symptoms are shown in Table 3. There was a significant mediation by total energy intake for the mindful eating domains Eating with Awareness and Eating without Distraction in the adjusted models 2 and 3 (indirect effects fully adjusted models: $B=-0.014,95 \% \mathrm{CI}-0.037,-0.002$ for Eating with Awareness; $B=-0.015,95 \%$ CI $-0.036,-0.002$ for Eating without Distraction). There was however no mediation by total energy intake for the mindful eating domains Focused Eating and Eating in response to Hunger and Satiety Cues in all of the adjusted models (indirect effects fully adjusted models: $B=0.004,95 \%$ CI $-0.002,0.016$ for Focused Eating; $B=0.003,95 \%$ CI $-0.0003,0.011$ for Eating in response to Hunger and Satiety Cues).

In Fig. 3, the coefficients of the various paths of the mediation models with total energy intake as mediator are shown. A higher total energy intake was associated with more depressive symptoms when controlling for any of the mindful eating domains: an increase of $418.4 \mathrm{~kJ} / \mathrm{d}(100 \mathrm{kcal} / \mathrm{d})$ was associated with an increase of $0 \cdot 1$ points in the depressive symptoms score in the fully adjusted models. The mindful eating domains Eating with Awareness and Eating without Distraction were significantly associated with total energy intake: a 1 point increase in Eating with Awareness was associated with a decrease of 
Table 2 Pearson correlations between the mindful eating domains, food intake, depressive symptoms and descriptive characteristics among the study sample of Dutch people aged 55 years or above from the Longitudinal Aging Study Amsterdam ( $n$ 929)

\begin{tabular}{|c|c|c|c|c|c|c|c|c|c|c|c|}
\hline & 1 & 2 & 3 & 4 & 5 & 6 & 7 & 8 & 9 & 10 & 11 \\
\hline 1. Focused Eating & & $0 \cdot 218^{\star \star \star}$ & $0 \cdot 284^{\star \star \star}$ & $0.144^{\star \star *}$ & $0.250^{\star *}$ & 0.057 & $-0.174^{\star * *}$ & $-0.204^{\star \star *}$ & -0.046 & $-0 \cdot 159^{\star \star \star}$ & $-0.143^{\text {*** }}$ \\
\hline $\begin{array}{l}\text { 2. Eating in response } \\
\text { to Hunger and } \\
\text { Satiety Cues }\end{array}$ & & & -0.052 & $-0 \cdot 115^{\star \star \star}$ & $0.077^{*}$ & $0.073^{*}$ & 0.031 & 0.033 & -0.061 & $-0.075^{\star}$ & $-0.132^{\star \star \star}$ \\
\hline $\begin{array}{l}\text { 3. Eating with } \\
\text { Awareness }\end{array}$ & & & & $0.411^{\star *}$ & $0.085^{\star \star}$ & $-0.069^{*}$ & $-0.138^{\star \star \star}$ & $-0 \cdot 156^{\star \star \star}$ & -0.019 & 0.023 & $-0 \cdot 144^{\star \star \star}$ \\
\hline $\begin{array}{l}\text { 4. Eating without } \\
\text { Distraction }\end{array}$ & & & & & -0.033 & $-0.087^{\star \star}$ & $-0 \cdot 201^{\star \star \star}$ & $-0 \cdot 161^{\star \star \star}$ & -0.045 & $0.084^{*}$ & 0.018 \\
\hline 5. MDS & & & & & & $0.162^{* * *}$ & $-0.148^{\star * *}$ & $-0 \cdot 111^{\star \star \star}$ & -0.028 & $-0 \cdot 121^{\star \star *}$ & $-0.161^{\star \star \star}$ \\
\hline 6. Energy intake & & & & & & & -0.021 & 0.002 & $-0.383^{\star \star \star}$ & $-0.092^{\star \star \star}$ & $-0.072^{\star}$ \\
\hline $\begin{array}{l}\text { 7. Depressive } \\
\text { symptoms, } \\
\text { baseline }\end{array}$ & & & & & & & & $0.592^{\star \star \star}$ & $0 \cdot 157^{\star * *}$ & $0.091^{* *}$ & $0.091^{* *}$ \\
\hline $\begin{array}{l}\text { 8. Depressive } \\
\text { symptoms, } \\
\text { follow-up }\end{array}$ & & & & & & & & & $0 \cdot 153^{\star \star \star}$ & $0.204^{\star \star \star}$ & $0.079^{*}$ \\
\hline $\begin{array}{l}\text { 9. Sex } \\
\text { 10. Age } \\
\text { 11. BMI }\end{array}$ & & & & & & & & & & 0.017 & $\begin{array}{r}-0.060 \\
0.029\end{array}$ \\
\hline
\end{tabular}

MDS, Mediterranean Diet Score.

${ }^{\star} P<0.05,{ }^{\star *} P<0.01,{ }^{\star \star *} P<0.001$.
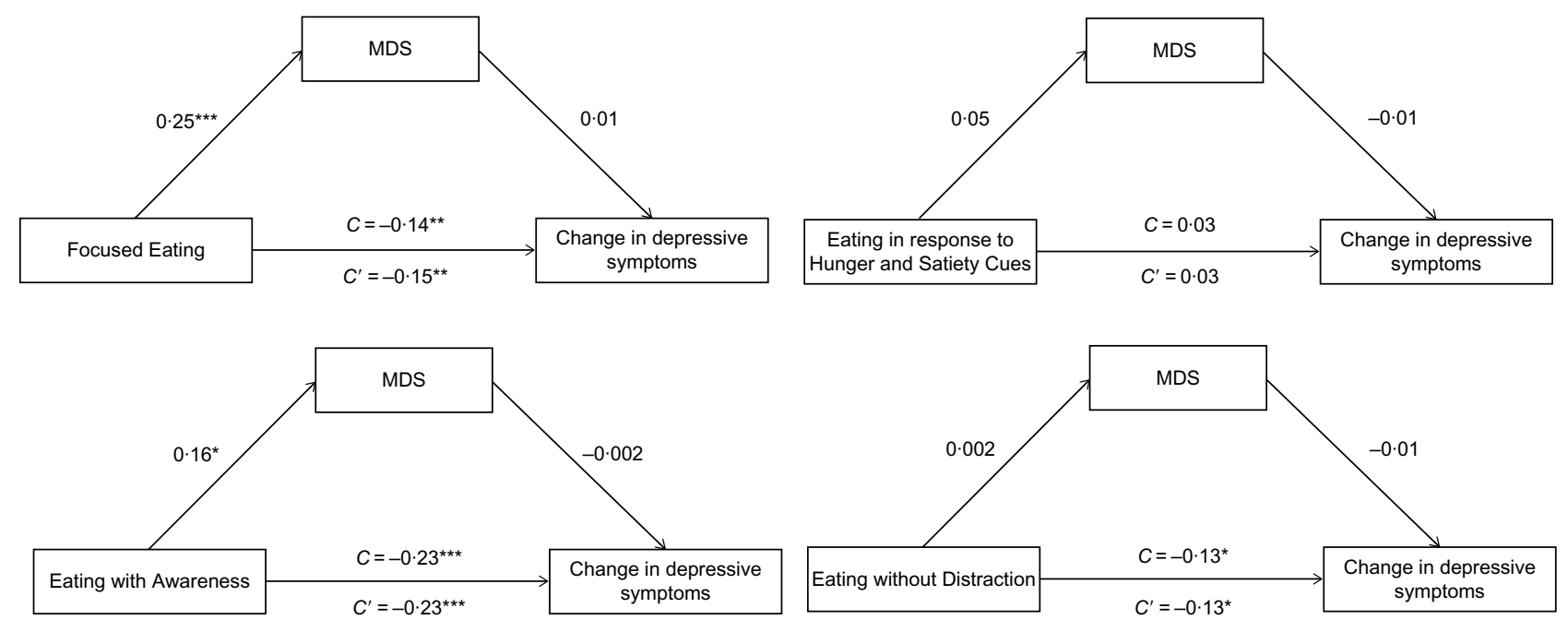

Fig. 2 Mediation models of the associations between four mindful eating domains and 3-year change in depressive symptoms via diet quality (Mediterranean Diet Score; MDS) in a sample of people aged 55 years or above from the Longitudinal Aging Study Amsterdam ( $n$ 929). Unstandardized regression coefficients from a bootstrap procedure with 5000 samples are provided along the paths. Analyses are adjusted for baseline depressive symptoms, sex, age, educational level, smoking status, physical activity level and total energy intake (model 3). $C$, total effect; $C^{\prime}$, direct effect; ${ }^{\star} P<0.05,{ }^{\star \star} P<0.01,{ }^{\star \star \star} P P 0.001$

$99.2 \mathrm{~kJ} / \mathrm{d}(23.7 \mathrm{kcal} / \mathrm{d})$, and a 1 point increase in Eating without Distraction was associated with a decrease of $105.4 \mathrm{~kJ} / \mathrm{d}$ $(25 \cdot 2 \mathrm{kcal} / \mathrm{d})$. Changes in associations when additionally adjusting for BMI (model 4) were negligible (e.g. indirect effect for Eating without Distraction model 4: $B=-0 \cdot 016$, $95 \%$ CI $-0.038,-0.003$; association between Eating without Distraction and energy intake model $4: B=-24.5)$. Inclusion of people with extreme values on energy intake ( $n$ 16) did not change any of these results. Results of the sensitivity analyses for the two significant mediation models can be found in the online supplementary material, Supplemental File S1.

\section{Post hoc analysis: multiple mediation}

Simple mediation analyses were conducted as quality and quantity were both independently associated with depression in previous studies and because there was only a small correlation between food quantity and quality in the current study $(r=0 \cdot 162$, see Table 2$)$. However, because of this correlation, multiple mediation analyses with the MDS and total energy intake as parallel mediators were conducted as post hoc analyses. By testing multiple mediators in one model compared with simple mediation models, the likelihood of parameter bias due to omitted variables is reduced ${ }^{(55)}$. A significant total indirect effect 

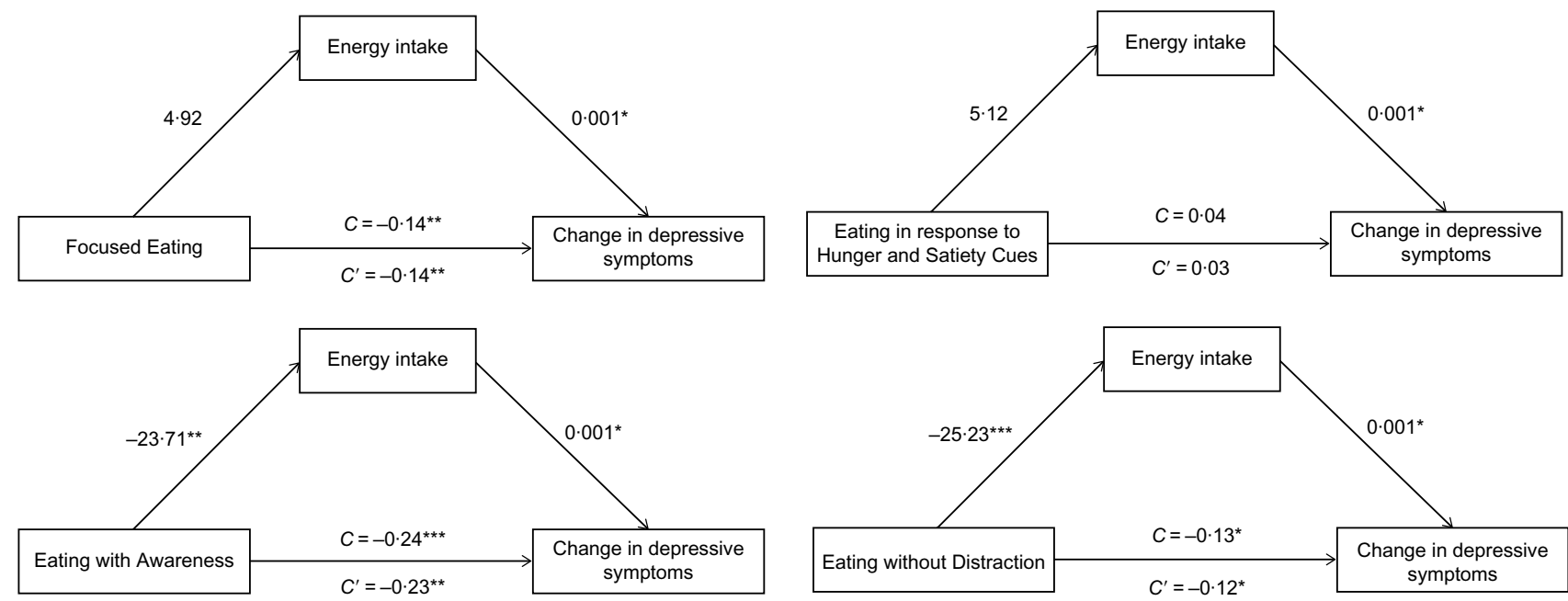

Fig. 3 Mediation models of the associations between four mindful eating domains and 3-year change in depressive symptoms via diet quantity (total energy intake; $\mathrm{kcal} / \mathrm{d} ; 1 \mathrm{kcal}=4.184 \mathrm{~kJ}$ ) in a sample of people aged 55 years or above from the Longitudinal Aging Study Amsterdam ( $n$ 929). Unstandardized regression coefficients from a bootstrap procedure with 5000 samples are provided along the paths. Analyses are adjusted for baseline depressive symptoms, sex, age, educational level, smoking status, physical activity level and alcohol consumption (model 3). $C$, total effect; $C^{\prime}$, direct effect; ${ }^{\star} P<0.05,{ }^{\star \star} P<0.01,{ }^{\star * \star} P<0.001$

Table 3 Indirect effects from mediation analyses of diet quality (Mediterranean Diet Score; MDS) and diet quantity (total energy intake; $\mathrm{kcal} / \mathrm{d} ; 1 \mathrm{kcal}=4.184 \mathrm{~kJ}$ ) in the associations of four mindful eating domains with 3-year change in depressive symptoms in a sample of people aged 55 years or above from the Longitudinal Aging Study Amsterdam ( $n 929)$

\begin{tabular}{|c|c|c|c|c|c|c|}
\hline & \multicolumn{3}{|c|}{ MDS } & \multicolumn{3}{|c|}{ Total energy intake } \\
\hline & $B$ & $\begin{array}{l}\text { Lower bootstrap } \\
95 \% \mathrm{Cl}\end{array}$ & $\begin{array}{c}\text { Upper bootstrap } \\
95 \% \mathrm{Cl}\end{array}$ & $B$ & $\begin{array}{c}\text { Lower bootstrap } \\
95 \% \mathrm{Cl}\end{array}$ & $\begin{array}{c}\text { Upper bootstrap } \\
95 \% \mathrm{Cl}\end{array}$ \\
\hline \multicolumn{7}{|c|}{ Focused Eating } \\
\hline Model 1 & -0.0002 & -0.025 & 0.023 & 0.002 & -0.002 & 0.012 \\
\hline Model 2 & 0.006 & -0.011 & 0.026 & 0.005 & -0.001 & 0.017 \\
\hline Model 3 & 0.002 & -0.015 & 0.021 & 0.004 & -0.002 & 0.016 \\
\hline \multicolumn{7}{|c|}{ Eating in response to Hunger and Satiety Cues } \\
\hline Model 1 & -0.002 & -0.010 & 0.002 & 0.001 & -0.003 & 0.007 \\
\hline Model 2 & 0.000 & -0.004 & 0.005 & 0.003 & -0.0004 & 0.010 \\
\hline Model 3 & -0.001 & -0.006 & 0.003 & 0.003 & -0.0003 & 0.011 \\
\hline \multicolumn{7}{|c|}{ Eating with Awareness } \\
\hline Model 1 & -0.004 & -0.020 & 0.005 & -0.002 & -0.018 & 0.008 \\
\hline Model 2 & 0.002 & -0.009 & 0.018 & -0.012 & -0.035 & -0.001 \\
\hline Model 3 & 0.0001 & -0.016 & 0.015 & -0.014 & -0.037 & -0.002 \\
\hline \multicolumn{7}{|c|}{ Eating without Distraction } \\
\hline Model 1 & 0.004 & -0.003 & 0.019 & -0.002 & -0.016 & 0.008 \\
\hline Model 2 & -0.0001 & -0.007 & 0.004 & -0.013 & -0.033 & -0.001 \\
\hline Model 3 & 0.001 & -0.004 & 0.005 & -0.015 & -0.036 & -0.002 \\
\hline
\end{tabular}

Depressive symptoms are measured with the Center for Epidemiologic Studies Depression Scale continuous score. Unstandardized regression coefficients ( $B$ ) and percentile bootstrap confidence intervals are reported (bootstrap sample size $=5000$ ). Bold values indicate statistically significant results.

Model 1 was adjusted for baseline depressive symptoms; model 2 was additionally adjusted for sex, age, educational level, smoking status and physical activity level; model 3 (MDS) was additionally adjusted for total energy intake; model 3 (total energy intake) was additionally adjusted for alcohol consumption.

is no prerequisite for investigating specific indirect effects; either or both types of effect are interesting and worth investigating ${ }^{(55)}$. Therefore, both the total indirect effect of the set of mediators as well as the specific indirect effects of each individual mediator conditional on the other mediator in the model were reported, together with the corresponding percentile $95 \%$ confidence intervals. It is important to note that the specific indirect effect through either quality or quantity of food intake represents the ability of that variable to mediate the association of mindful eating with depressive symptoms conditional on the inclusion of the other variable in the model: it is thus the unique mediating effect of that variable.

To test whether MDS and total energy intake mediated the associations of mindful eating domains with change in depressive symptoms, percentile bootstrap confidence intervals for the indirect effects were calculated in multiple mediation models. The total and specific indirect effects of 
the multiple mediation analyses of food quality and food quantity in the associations of the mindful eating domains with depressive symptoms are shown in the online supplemental material, Supplemental File S2. No mediation by food intake was found for the domains Focused Eating (total mediation: $B=0.006,95 \% \mathrm{CI}-0.014,0.025$ ) and Eating in response to Hunger and Satiety Cues (total mediation: $B=0.002$, $95 \%$ CI $-0.004,0.009)$. For the domain Eating with Awareness, only specific mediation by total energy intake was found in the fully adjusted model ( $B=-0.018$, 95\% CI -0.039 , -0.001). For the domain Eating without Distraction, both total mediation and specific mediation by total energy intake were found in the fully adjusted model (indirect effect: $B=-0 \cdot 016$, $95 \% \mathrm{CI}-0.036,-0.001$ for total mediation; $B=-0.017$, $95 \%$ CI $-0.036,-0.002$ for mediation by total energy intake). As these results are similar to the results of the simple mediation analyses, we can conclude that the results of these simple mediation analyses were not biased due to the omitted variable (either food quality or food quantity).

\section{Discussion}

The present study is the first to investigate both quality and quantity of food intake as underlying mechanisms in the associations between mindful eating domains and change in depressive symptoms. In the study, higher scores on the mindful eating domains Eating with Awareness and Eating without Distraction were associated with a decrease in depressive symptoms after 3 years through lower total energy intake. No mediation was observed for the domains Focused Eating and Eating in response to Hunger and Satiety Cues.

Regarding diet quality as a potential underlying mechanism, we found that diet quality was no mediator between the mindful eating domains and change in depressive symptoms. This is likely caused by the non-significant associations between the MDS and change in depressive symptoms when controlling for the mindful eating domains, which is in contrast to recent meta-analyses regarding diet quality and (incidence of) depressive symptoms. In these meta-analyses, adherence to a Mediterranean diet was associated with a lower risk of depressive symptoms over time ${ }^{(33-35)}$. A review of longitudinal studies among older adults showed mixed findings ${ }^{(56)}$. Gougeon et al. also found in a community-dwelling older population that dietary patterns were not related to depression, whereas energy intake was $^{(14)}$. It might thus be that quantity of food intake, instead of quality of food intake, becomes more important in explaining changes in depression over time in older adults.

Although the MDS was not a mediating factor in the associations between mindful eating and depression, higher scores on the domains Focused Eating and Eating with Awareness were associated with a higher MDS, indicative of a healthier diet. These results are in concordance with a study showing that higher attentive eating was associated with a higher intake of fruit and vegetables in pregnant women ${ }^{(23)}$, as well as with an experimental study showing that higher general mindfulness was related to more often choosing fruit instead of unhealthy options ${ }^{(57)}$. Although there is no indication that a better diet quality is related to depression in our study, mindful eating might be beneficial in increasing general health status through increased diet quality. Further research is needed to investigate this.

Regarding diet quantity as a potential underlying mechanism, we found that energy intake mediated associations of two mindful eating domains with depression: higher scores on Eating with Awareness and Eating without Distraction were related to lower energy intake per day, which was associated with fewer depressive symptoms. That controlling the analyses for physical activity and BMI did not change any of the conclusions suggests that energy intake is uniquely associated with mindful eating, independent of BMI and physical activity. Previous studies on distracted eating found that listening to a story ${ }^{(58,59)}$, watching television ${ }^{(59)}$ and listening to music ${ }^{(60)}$ were associated with higher energy intake. As mechanisms of action for the associations between mindful eating and food intake are still unclear, future research could examine some of these potential mechanisms, such as reduced automaticity, increased prioritization of joy, increased interoceptive awareness, increased self-regulation or decreases in food cravings.

Energy intake was not a mediator in the associations of Focused Eating and Eating in response to Hunger and Satiety Cues with change in depressive symptoms. Both Focused Eating and Eating in response to Hunger and Satiety Cues were not significantly associated with total energy intake. That we did not find an association between Focused Eating and total energy intake is in concordance with studies not showing associations between attentive eating and direct food intake ${ }^{(18)}$ and with some recent experimental studies that did not find reduced later amounts of intake ${ }^{(20,21)}$, but is contradictory to other studies that did find these associations with later food intake ${ }^{(18,19)}$. It could however be that although higher Focused Eating potentially leads to reduced later amounts of food intake, this is compensated for at other time points, thereby leading to the same amount of total energy consumed in a daily dietary pattern. Energy intake measured after experimental manipulations might not be characteristic of habitual total energy intake. That we did not find any association with the domain Eating in response to Hunger and Satiety Cues might be due to the older age of the sample: changes in hunger, satiety and appetite occur due to the ageing process $^{(61)}$.

Strengths of the present study are the use of data on both diet quality and quantity and the large national representative sample of Dutch middle-aged and older adults, whereas previous studies investigated specific dietary 
aspects and mostly used students and female samples. A limitation of this sample is that our results might not be generalizable to younger age samples. Food intake might change during ageing; although a higher energy intake is generally associated with depression, lower energy intake may also be predictive of depression due to declining general health in older adults ${ }^{(14)}$. However, in the present study, we found that a higher energy intake was associated with more depressive symptoms over time. Another limitation is that mindful eating and food intake were measured at the same time point, whereas in mediation analyses desirably the temporal order of all of the variables is tested. Although the causal order of the mindful eating domains and food intake therefore cannot be established, mindful eating is hypothesized to precede food intake in time. Another limitation regarding the temporality is that mindful eating and food intake were measured in-between the baseline and follow-up measures of depressive symptoms. As a result, depressive symptoms measured at baseline might have influenced mindful eating and food intake measured in the ancillary study. Due to these limitations, the current mediation analyses are only exploratory. Our results provide more insight into possible mechanisms underlying the associations between mindful eating and depression and may generate hypotheses for further study. Further research is needed to test the associations of mindful eating with change in food intake over time and ultimately with change in depressive symptoms.

As food quality mediated only the associations of Eating with Awareness and Eating without Distraction with change in depressive symptoms, further research should investigate other potential underlying mechanisms. In a previous study we found that the psychological eating styles, especially external eating, mediated the association between mindful eating and change in depressive symptoms ${ }^{(13)}$. Another potential mediating variable is obesity, as obesity is bidirectionally linked with depression: the presence of one increases the risk for developing the other $^{(9,62)}$. General mindfulness interventions were effective in reducing weight ${ }^{(63)}$. More research into associations between mindful eating and BMI, as well as BMI as mediating factor in the associations between mindful eating and depression, would be very interesting.

In the present study, higher scores on two mindful eating domains were associated with a decrease in depressive symptoms through lower total energy intake. Diet quantity, but not diet quality, could be a possible underlying mechanism in the associations between mindful eating and change in depressive symptoms.

\section{Acknowledgements}

Financial support: This work was supported by the European Union FP7 MooDFOOD Project 'Multi-country
cOllaborative project on the rOle of Diet, FOod-related behaviour, and Obesity in the prevention of Depression' (grant agreement number 613598). The Longitudinal Aging Study Amsterdam is supported by a grant from the Netherlands Ministry of Health Welfare and Sports, Directorate of Long-Term Care. The data collection in 2012-2013 was financially supported by the Netherlands Organization for Scientific Research (NWO) in the framework of the project 'New Cohorts of young old in the 21st century' (file number 480-10-014). The funding agencies had no role in the design, analysis or writing of this article. Conflict of interest: The authors declare no conflict of interest. Authorship: L.H.H.W., M.V., I.A.B. and T.v.S. designed the study. L.H.H.W. and L.E.M.E. collected the data. L.H.H.W. wrote the first draft with contributions from M.V., I.A.B. and T.v.S. L.H.H.W. conducted the statistical analyses. All authors reviewed and commented on subsequent drafts of the manuscript. All authors approved the final version. Ethics of buman subject participation: This study was conducted according to the guidelines laid down in the Declaration of Helsinki and all procedures involving human subjects in LASA were approved by the Medical Ethics Committee of the VU University Medical Center Amsterdam. All participants provided written informed consent.

\section{Supplementary material}

To view supplementary material for this article, please visit https://doi.org/10.1017/S1368980019003732

\section{References}

1. World Health Organization (2018) Depression factsheet. https://www.who.int/en/news-room/fact-sheets/detail/ depression (accessed September 2018).

2. Hedman-Lagerlöf M, Hedman-Lagerlöf E \& Öst L-G (2018) The empirical support for mindfulness-based interventions for common psychiatric disorders: a systematic review and meta-analysis. Psychol Med 48, 2116-2129.

3. Strauss C, Cavanagh K, Oliver A et al. (2014) Mindfulnessbased interventions for people diagnosed with a current episode of an anxiety or depressive disorder: a meta-analysis of randomised controlled trials. PLoS One 9, e96110.

4. Kuyken W, Hayes R, Barrett B et al. (2015) Effectiveness and cost-effectiveness of mindfulness-based cognitive therapy compared with maintenance antidepressant treatment in the prevention of depressive relapse or recurrence (PREVENT): a randomised controlled trial. Lancet 386, 63-73.

5. Khoury B, Sharma M, Rush SE et al. (2015) Mindfulnessbased stress reduction for healthy individuals: a metaanalysis. J Psychosom Res 78, 519-528.

6. Cabout M, Brouwer I, Visser M et al. (2017) The MooDFOOD project: prevention of depression through nutritional strategies. Nutr Bull 42, 94-103.

7. Firth J, Marx W, Dash S et al. (2019) The effects of dietary improvement on symptoms of depression and anxiety: a meta-analysis of randomized controlled trials. Psychosom Med 81, 265-280. 
8. Jacka FN (2017) Nutritional psychiatry: where to next? EBioMedicine 17, 24-29.

9. Milaneschi Y, Simmons WK, Rossum EF et al. (2019) Depression and obesity: evidence of shared biological mechanisms. Mol Psychiatry 24, 18-33.

10. Warren JM, Smith N \& Ashwell M (2017) A structured literature review on the role of mindfulness, mindful eating and intuitive eating in changing eating behaviours: effectiveness and associated potential mechanisms. Nutr Res Rev 30, 272-283.

11. O'Reilly GA, Cook L, Spruijt-Metz D et al. (2014) Mindfulnessbased interventions for obesity-related eating behaviours: a literature review. Obes Rev 15, 453-461.

12. Winkens LHH, van Strien T, Brouwer IA et al. (2018) Associations of mindful eating domains with depressive symptoms and depression in three European countries. J Affect Disord 228, 26-32.

13. Winkens LHH, van Strien T, Brouwer IA et al. (2019) Mindful eating and change in depressive symptoms: mediation by psychological eating styles. Appetite 133, 204-211.

14. Gougeon L, Payette H, Morais J et al. (2015) Dietary patterns and incidence of depression in a cohort of communitydwelling older Canadians. J Nutr Health Aging 19, 431-436.

15. Lopresti AL, Hood SD \& Drummond PD (2013) A review of lifestyle factors that contribute to important pathways associated with major depression: diet, sleep and exercise. J Affect Disord 148, 12-27.

16. Winter G, Hart RA, Charlesworth RP et al. (2018) Gut microbiome and depression: what we know and what we need to know. Rev Neurosci 29, 629-643.

17. Beshara M, Hutchinson AD \& Wilson C (2013) Does mindfulness matter? Everyday mindfulness, mindful eating and self-reported serving size of energy dense foods among a sample of South Australian adults. Appetite 67, $25-29$.

18. Tapper K (2017) Can mindfulness influence weight management related eating behaviors? If so, how? Clin Psychol Rev 53, 122-134.

19. Seguias L \& Tapper K (2018) The effect of mindful eating on subsequent intake of a high calorie snack. Appetite 121, 93-100.

20. Whitelock V, Gaglione A, Davies-Owen J et al. (2019) Focused attention during eating enhanced memory for meal satiety but did not reduce later snack intake in men: a randomised within-subjects laboratory experiment. Appetite 136, 124-129.

21. Whitelock V, Higgs S, Brunstrom JM et al. (2018) No effect of focused attention whilst eating on later snack food intake: two laboratory experiments. Appetite 128, 188-196.

22. Miller CK, Kristeller JL, Headings A et al. (2014) Comparison of a mindful eating intervention to a diabetes selfmanagement intervention among adults with type 2 diabetes: a randomized controlled trial. Health Educ Behav 41, 145-154.

23. Hutchinson AD, Charters M, Prichard I et al. (2017) Understanding maternal dietary choices during pregnancy: the role of social norms and mindful eating. Appetite 112, 227-234.

24. Herbert BM, Blechert J, Hautzinger M et al. (2013) Intuitive eating is associated with interoceptive sensitivity. Effects on body mass index. Appetite 70, 22-30.

25. Herbert BM \& Pollatos O (2014) Attenuated interoceptive sensitivity in overweight and obese individuals. Eat Behav 15, 445-448.

26. Tapper K (2018) Mindfulness and craving: effects and mechanisms. Clin Psychol Rev 59, 101-117.

27. Paolini B, Burdette JH, Laurienti PJ et al. (2012) Coping with brief periods of food restriction: mindfulness matters. Front Aging Neurosci $\mathbf{4}, 13$.
28. Chao A, Grilo CM, White MA et al. (2014) Food cravings, food intake, and weight status in a community-based sample. Eat Behav 15, 478-482.

29. Kristeller JL \& Wolever RQ (2010) Mindfulness-based eating awareness training for treating binge eating disorder: the conceptual foundation. Eat Disord 19, 49-61.

30. Hu FB (2002) Dietary pattern analysis: a new direction in nutritional epidemiology. Curr Opin Lipidol 13, 3-9.

31. Sofi F, Cesari F, Abbate R et al. (2008) Adherence to Mediterranean diet and health status: meta-analysis. BMJ 337, a1344.

32. Ocké MC (2013) Evaluation of methodologies for assessing the overall diet: dietary quality scores and dietary pattern analysis. Proc Nutr Soc 72, 191-199.

33. Molendijk M, Molero P, Sánchez-Pedreño FO et al. (2018) Diet quality and depression risk: a systematic review and dose-response meta-analysis of prospective studies. J Affect Disord 226, 346-354.

34. Lassale C, Batty GD, Baghdadli A et al. (2019) Healthy dietary indices and risk of depressive outcomes: a systematic review and meta-analysis of observational studies. Mol Psychiatry 24, 965-986.

35. Nicolaou M, Colpo M, Vermeulen E et al. (2019) Association of a priori dietary patterns with depressive symptoms: a harmonized meta-analysis of observational studies. Psychol Med. Published online: 14 August 2019. doi: 10.1017/ S0033291719001958.

36. Mooreville M, Shomaker LB, Reina SA et al. (2014) Depressive symptoms and observed eating in youth. Appetite 75, 141-149.

37. Gougeon L, Payette H, Morais J et al. (2015) Dietary patterns and incidence of depression in a cohort of communitydwelling older Canadians. J Nutr Health Aging 19, 431-436.

38. Hoogendijk EO, Deeg DJH, Poppelaars J et al. (2016) The Longitudinal Aging Study Amsterdam: cohort update 2016 and major findings. Eur J Epidemiol 31, 927-945.

39. Huisman M, Poppelaars J, van der Horst M et al. (2011) Cohort profile: the Longitudinal Aging Study Amsterdam. Int J Epidemiol 40, 868-876.

40. Winkens LHH, van Strien T, Barrada JR et al. (2018) The mindful eating behavior scale: development and psychometric properties in a sample of Dutch adults aged 55 years and older. J Acad Nutr Diet 118, 1277-1290.

41. Radloff LS (1977) The CES-D scale: a self-report depression scale for research in the general population. Appl Psychol Meas 1, 385-401.

42. World Health Organization Collaborating Centre for Drug Statistics Methodology (2018) Guidelines for ATC Classification and DDD Assignment. https://www.whocc. no/atc_ddd_index/ (accessed September 2018).

43. Beukers MH, Dekker LH, de Boer EJ et al. (2015) Development of the HELIUS food frequency questionnaires: ethnic-specific questionnaires to assess the diet of a multiethnic population in The Netherlands. Eur J Clin Nutr 69, 579-584.

44. Siebelink E, Geelen A, de Vries JH (2011) Self-reported energy intake by FFQ compared with actual energy intake to maintain body weight in 516 adults. Br J Nutr 106, 274-281.

45. Stichting NEVO (2011) NEVO-tabel 2011: NEVO (Nederlands Voedingsstoffenbestand). Zeist: Stichting NEVO.

46. Trichopoulou A, Costacou T, Bamia C et al. (2003) Adherence to a Mediterranean diet and survival in a Greek population. New Engl J Med 348, 2599-2608.

47. Panagiotakos DB, Pitsavos C, Arvaniti F et al. (2007) Adherence to the Mediterranean food pattern predicts the prevalence of hypertension, hypercholesterolemia, diabetes and obesity, among healthy adults; the accuracy of the MedDietScore. Prev Med 44, 335-340.

48. Bonaccio M, Di Castelnuovo A, Costanzo S et al. (2018) Mediterranean diet and mortality in the elderly: a prospective cohort study and a meta-analysis. Br J Nutr 120, 841-854. 
49. Stel VS, Smit JH, Pluijm SMF et al. (2004) Comparison of the LASA physical activity questionnaire with a 7-day diary and pedometer. J Clin Epidemiol 57, 252-258.

50. Central Bureau of Statistics (1989) Health Interview Questionnaire. The Hague: Central Bureau of Statistics.

51. Willett W (1998) Issues in analysis and presentation of dietary data. In Nutritional Epidemiology, pp. 321-346. New York: Oxford University Press.

52. Hayes A (2013) Introduction to Mediation, Moderation, and Conditional Process Analysis. A Regression-Based Approach. New York: Guilford.

53. Tingley D, Yamamoto T, Hirose K et al. (2014) Mediation: R package for causal mediation analysis. J Stat Softw 59, issue 5, doi: 10.18637/jss.v059.i05.

54. Imai K, Keele L \& Tingley D (2010) A general approach to causal mediation analysis. Psychol Methods 15, 309-334.

55. Preacher KJ \& Hayes AF (2008) Asymptotic and resampling strategies for assessing and comparing indirect effects in multiple mediator models. Behav Res Methods 40, 879-891.

56. Gougeon L (2016) Dietary patterns and depression in community-dwelling older adults: state of the evidence. Curr Nutr Rep 5, 106-112.
57. Jordan CH, Wang W, Donatoni L et al. (2014) Mindful eating: trait and state mindfulness predict healthier eating behavior. Pers Individ Dif 68, 107-111.

58. Long S, Meyer C, Leung N et al. (2011) Effects of distraction and focused attention on actual and perceived food intake in females with non-clinical eating psychopathology. Appetite 56, 350-356.

59. Bellisle F, Dalix A \& Slama G (2004) Non food-related environmental stimuli induce increased meal intake in healthy women: comparison of television viewing versus listening to a recorded story in laboratory settings. Appetite 43, 175-180.

60. Stroebele N \& de Castro JM (2006) Listening to music while eating is related to increases in people's food intake and meal duration. Appetite 47, 285-289.

61. Elsner RJ (2002) Changes in eating behavior during the aging process. Eat Behav 3, 15-43.

62. Faith M, Butryn M, Wadden T et al. (2011) Evidence for prospective associations among depression and obesity in population-based studies. Obes Rev 12, e438-e453.

63. Carrière K, Khoury B, Günak M et al. (2018) Mindfulnessbased interventions for weight loss: a systematic review and meta-analysis. Obes Rev 19, 164-177. 